\title{
PREPARACIÓN DE FILTROS DE ADSORCIÓN A BASE DE ARCILLAS MODIFICADAS
}

\section{PREPARATION OF FILTERS ADSORPTION-BASED MODIFIED CLAYS}

\section{RESUMEN}

\author{
María del Rosario Sun Kou ${ }^{\mathrm{a}}$, José Inga, Abel Gutarra ${ }^{\mathrm{b}}$
}

La cantidad de contaminantes textiles (colorantes, surfactantes, fenoles entre otros) se ha incrementado en los últimos años en el Perú debido al aumento en los volúmenes de exportación, La adsorción es uno de los métodos más ampliamente usados para minimizar el grado de contaminación en los efluentes industriales, esto a motivado el desarrollo de materiales abundantes y de bajo costo que puedan ser utilizados como adsorbentes.

Para este trabajo se ha empleado bentonitas peruanas, sódicas (LI-01 y LI-03) y cálcicas (HU-01 y HU-02), modificadas por termoactivación con $\mathrm{HCl}$ y $\mathrm{H}_{2} \mathrm{SO}_{4}$. Estos materiales fueron probados en la adsorción de diferentes colorantes: Rojo 80, Rhodamine $\mathrm{B}$, rojo ácido $\mathrm{G}$, azul de metileno. Comparada con la arcilla natural, la muestra termoactivada LI-03 incrementó su poder de adsorción en 13 veces, mientras la muestra HU-01 el incremento fue de 11 veces. El proceso de adsorción se produjo a pH entre 3 y 4 y cuando fue adicionado electrolitos a la solución coloreada.El mejor método encontrado para la preparación de los filtros de adsorción a base de arcillas termoactivadas fue el de la esponja polimérica con tratamientos térmicos entre 500 a $980^{\circ} \mathrm{C}$. Los filtros preparados adsorbieron el $100 \%$ del colorante Rhodamine B y del azul de metileno. La adsorción se logró en menor grado con los colorantes rojo 80 y rojo ácido G.

Palabras claves: adsorción, filtros de adsorción, arcillas termoactivadas, colorantes textiles

\section{ABTRACT}

During the last years in Perú the amount of textile contamination (dyes, surfactants, phenols among others) has increased due to the increase in export volumes.Adsorption is one of the methods most often used to minimize the grade of contamination in industrial effluents, and it has encouraged the development of abundant materials wich are low cost and so they could be used as adsorbents.

For this work has been used peruvian bentonites, sodium (LI-01 and LI-03) and calcium (HU-01 and HU-02), modified by thermo-activation with $\mathrm{HCl}$ and $\mathrm{H}_{2} \mathrm{SO}_{4}$. These materials were tested in the adsorption of several dyes: Red 80, Rhodamine B, Acid Red G, methylene blue. A sample of thermo-activated LI-03 increased its power of adsorption thirteen times compared with the natural clays, on the other hand in the case of $\mathrm{HU}-01$ increase was eleven times. The adsorption process is produced in $\mathrm{pH}$ range of $3-4$ when electrolytes were added into dye solution. The best method found for the preparation of filters of adsorption from thermo-activated clays was the polymer sponge with thermic treatment in the range of $500-980^{\circ} \mathrm{C}$. Prepared filters adsorbed $100 \%$ of dyes: Rhodamine B and Methylene Blue. The adsorption got in low degree with Red dye 80 and Acid Red G.

Key words: Adsorption, filters of adsorption, thermo-activated clays, textile dyes.

\section{INTRODUCCION}

En el Perú la industria textil es una de las actividades económicas más importantes pero también una de las principales fuentes de contaminación de las aguas lacustres, fluviales y marítimas. Se ha encontrado que las sustancias que le confieren coloración a las aguas provenientes de la industria textil pueden tener un efecto inhibidor sobre la fotosíntesis. Además, en las corrientes naturales la degradación incompleta de las moléculas de colorante puede generar compuestos más tóxicos que los colorantes mismos y afectar así a los sistemas acuáticos.

El tratamiento de los efluentes textiles es particularmente complejo debido a los múltiples componentes que lo constituyen (además de los colorantes, los efluentes pueden contener mordientes que son sales metálicas que se utilizan durante el proceso de teñido, tensoactivos o surfactantes, fenoles y otros compuestos químicos específicos del proceso de teñido y acondicionamiento de las fibras textiles) [1].

Los métodos usuales para el tratamiento de efluentes textiles son: biodegradación, floculación, osmosis inversa, tecnologías de oxidación avanzada y adsorción, siendo esta última la más aplicada por su versatilidad y bajo costo de aplicación [2-5].Por otro lado, la cantidad de estos efluentes se ha incrementado en los últimos años en el Perú, debido al aumento en los volúmenes de exportación. Esto ha motivado el desarrollo de materiales abundantes y de bajo costo, para ser empleados como adsorbentes de contaminantes orgánicos e inorgánicos. En este trabajo se utilizó el método de adsorción, empleando como adsorbente un recurso no metálico de gran abundancia en nuestro país como son las arcillas esmectíticas, las cuales muestran en estado natural buenas pero limitadas propiedades como adsorbentes. Estudios realizados anteriormente [6] indican que se logra potenciar dichas propiedades mediante la modificación química, estructural y textural de las arcillas ya sea por activación termoácida o por intercambio ionico con sales de amonio. El objetivo de este trabajo fue la preparación de filtros de adsorción a base de estas arcillas modificadas, en los que se busca que posean una buena resistencia térmica y mecánica, y que mantengan sus propiedades adsorbentes, en la retención de los colorantes contenidos en los efluentes textiles. Por otro lado, dado que los materiales cerámicos porosos abarcan un amplio campo de aplicaciones en la industria, este estudio contribuye a la producción de filtros cerámicos 
en el Perú, utilizando una materia prima nacional como es la arcilla.

\section{MATERIALES Y MÉTODOS}

\section{Materiales}

a) Arcilla: Para este trabajo se recolectaron muestras de arcilla procedentes de Arequipa, Trujillo, Junín, Puno, Piura y Cajamarca, las cuales fueron sometidas a diferentes pruebas preliminares (tiempo de sedimentación, grado de hidratación, $\mathrm{pH}$, presencia de carbonatos). Dichos ensayos permitieron seleccionar las mejores arcillas, todas de la familia de las esmectitas, dos sódicas: LI-01, LI-03 y dos cálcicas: HU-01, HU-02.

b) Efluente: $\mathrm{Se}$ recolectaron cuatro muestras de efluentes textiles proporcionadas por una empresa textil ubicada en Huancayo, la cual trabaja con lana de oveja, llama y alpaca, detectándose presencia de surfactantes y colorantes en todos los efluentes, y solo se determinó presencia de cromo (III) en el efluente 2, obtenido a la salida del teñido en autoclave. Los ensayos experimentales con los filtros se realizó con el efluente denominado M4 que contiene en gran proporción el colorante textil conocido como Rhodamine B (índice de color: 45710), y en menor proporción se encontró el colorante rojo 80 (índice de color 35780) y el rojo ácido G. Adicionalmente, otros ensayos de adsorción se realizaron con el azul de metileno.

\section{Caracterización}

Las variaciones estructurales de los materiales fue evaluado por las técnicas de difracción de rayos $X(D R X)$, termo-gravimetría (ATG), espectroscopia infrarroja (FTIR) y microscopia electrónica de barrido (SEM-EDX).

\section{Preparación de los filtros de arcilla. Método de la esponja polimérica}

Este método consiste en la impregnación (en etapas sucesivas) de una esponja polimérica con una suspensión cerámica, seguido de un tratamiento térmico para quemar el polímero y finalizando con la sinterización del filtro cerámico formado [7].

La suspensión cerámica fue preparada a partir de una cantidad determinada de arcilla purificada (fracción montmorillonítica $\leq 2 \mu \mathrm{m}$ obtenida por sedimentación controlada de una suspensión de arcilla), dispersa en un medio líquido (por lo general agua destilada), a la cual se le agregó como aditivos, agentes enlazantes y dispersantes. El enlazante es un aditivo que modifica la viscosidad de la suspensión cerámica, además proporciona resistencia mecánica para su manipulación. El agente dispersante se adiciona para que las partículas de arcilla no precipiten, estabilizando la suspensión cerámica.

En el proceso seguido para la preparación de los filtros, se partió con una determinada dispersión de arcilla a la cual se agregó carboximetil celulosa de sodio (CMC) como enlazante en proporción de 0,1-2,5 \% en peso y poliacrilato de sodio (SOKALAN AS5) como dispersante en proporción de $0,5-1,5 \%$ en peso. La pasta viscosa formada por la suspensión de arcilla se impregnó a la esponja por medio de sucesivas operaciones de comprensión-expansión. La esponja impregnada se dejó secar durante $24 \mathrm{~h}$. Luego del secado se procedió a la calcinación de la esponja y de los aditivos, utilizando una velocidad de calentamiento de $2^{\circ} \mathrm{C} / \mathrm{min}$, hasta alcanzar una temperatura predeterminada y manteniendo dicha temperatura por un periodo de $3 \mathrm{~h}$.

\section{Activación de los filtros}

Para realizar la activación de los filtros, se diseño un sistema el cual se muestra en la figura 1.

Los filtros fueron puestos en contacto con una solución de ácido clorhídrico $2 \mathrm{~N}$, en una relación de $30 \mathrm{~mL}$ de ácido/g de arcilla, haciendo circular el ácido por los filtros de arcilla. Para mantener la temperatura del ácido a $90^{\circ} \mathrm{C}$, se utilizó un flujo de aceite que estuvo a $100^{\circ} \mathrm{C}$ aproximadamente, este tratamiento fue realizado por 2 horas. Luego del cual, se lavaron los filtros hasta la eliminación total de cloruros y se secó en estufa a $50 \stackrel{\circ}{\circ} \mathrm{C}$ por 16 horas.

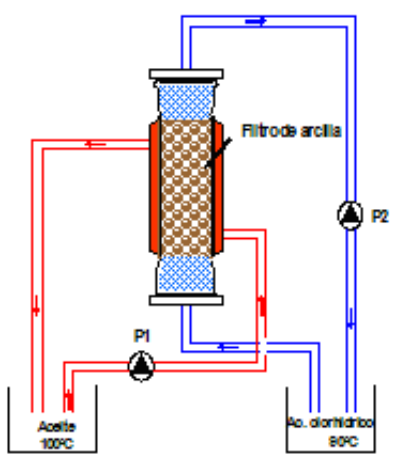

Figura 1. Sistema de activación para los filtros

\section{RESULTADOS}

En los difractogramas de la figura 2, se muestran algunas de las reflexiones identificadas como montmorillonita (M), muscovita $(\mathrm{Mu})$ y cristobalita (Cris). Se observa que la reflexión a $62.7^{\circ}$ (plano 060) de la montmorillonita permanece hasta los $600^{\circ} \mathrm{C}$, sin embargo el pico a 5.9 (plano 001) desaparece antes de los $200^{\circ} \mathrm{C}$. Para temperaturas mayores a $800^{\circ} \mathrm{C}$ aparece la fase mullita y la fase montmorillonita desaparece por completo.

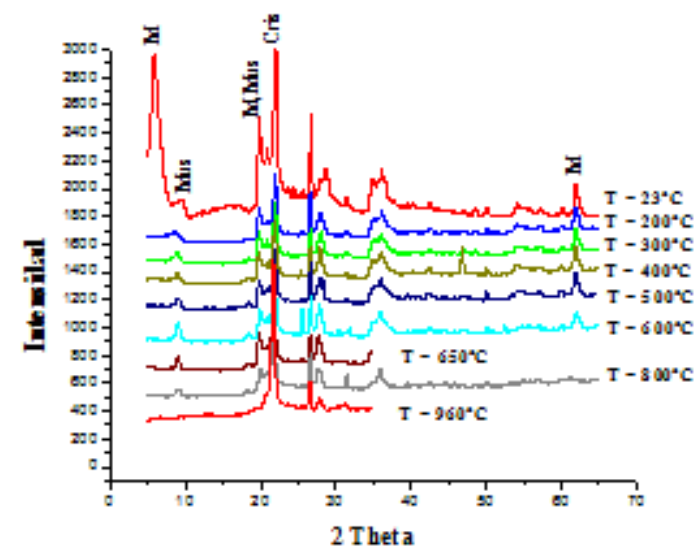

Figura 2. DRX de la arcilla esmectita tratada a diferentes temperaturas $(\mathrm{M}=$ mont-morillonita, $\mathrm{Mus}=$ muscovita, Cris $=$ cristobalita $)$

En la figura 3 se observan las curvas termogravimétricas de las muestras de arcilla en polvo. Las arcillas naturales 
y las activadas muestran un comportamiento similar. Tanto la prueba realizada en aire como en atmósfera de oxígeno muestran una pérdida de agua entre $50-100^{\circ} \mathrm{C}$ y la deshidroxilacion (pérdida de $\mathrm{OH}$ estructural) alrededor de $600^{\circ} \mathrm{C}[8]$.

Las pruebas realizadas por rayos $\mathrm{X}$ y la termografía nos indican que durante el calentamiento de los filtros se deben procesar las muestras a temperaturas que no sobrepasen los $600{ }^{\circ} \mathrm{C}$, para evitar la posible descomposición térmica de la arcilla y por lo tanto la pérdida de la capacidad de adsorción de los contaminantes.

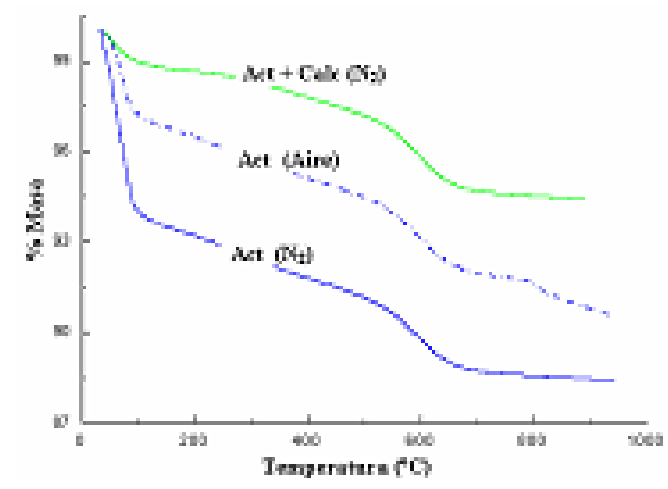

Figura 3. Curvas termogravimétricas de la arcilla activada calcinada en atmósfera de aire y nitrógeno

Para la preparación de los filtros, se realizaron diversas pruebas como se muestra en la tabla 1, variando los componentes de la suspensión cerámica así como del tratamiento térmico para su conformación.

Los filtros cerámicos con buena porosidad y resistencia mecánica se obtuvieron con los parámetros de las dos últimas muestras [7 y 8] de la tabla 1.

Tabla 1. Mezclas hechas para la preparación de la suspensión cerámica

\begin{tabular}{|c|c|c|c|c|c|c|}
\hline $\mathrm{N}^{\circ}$ & $\begin{array}{c}\text { Arcilla } \\
(\mathrm{g})\end{array}$ & $\begin{array}{c}\text { Agua } \\
(\mathrm{mL})\end{array}$ & $\begin{array}{c}\text { Enla- } \\
\text { zante } \\
(\mathrm{g})\end{array}$ & $\begin{array}{c}\text { Disper- } \\
\mathbf{s a n t e} \\
(\mathrm{g})\end{array}$ & $\mathrm{T}^{\circ}{ }^{\circ} \mathrm{C}$ & $\mathrm{T}^{\circ} \mathrm{C}$ \\
\hline 1 & 8 & 8 & 0.216 & - & 500 & 750 \\
\hline 2 & 8 & 8 & 0.213 & - & 500 & 780 \\
\hline 3 & 8 & 12 & - & - & 500 & 590 \\
\hline 4 & 8 & 22 & 0.032 & 0.37 & 500 & 590 \\
\hline 5 & 8 & 20 & - & 0.185 & 500 & 590 \\
\hline 6 & 8 & 23.5 & - & 0.074 & 460 & 590 \\
\hline 7 & 8 & 65 & 0.175 & 0.925 & 460 & 590 \\
\hline 8 & 8 & 46 & 0.08 & 0.666 & 470 & 600 \\
\hline
\end{tabular}

En la figura 4 se observa una fotografía de los filtros hechos con geometría circular (diámetro $30 \mathrm{~mm}$ ) y su micrografías a 30 aumentos obtenido mediante el microscopio electrónico de barrido (SEM-EDX) modelo RJ LEE SV 20.

Se realizaron una serie de ensayos de adsorción de colorantes, a fin de evaluar los parámetros: contenido de los agentes dispersante y enlazante, masa de material, temperatura de calcinación y tiempo de calcinación, estos estudios permitieron establecer una relación entre la descomposición térmica de la arcilla y la pérdida de la capacidad de adsorción de los contaminantes, en este caso los colorantes.
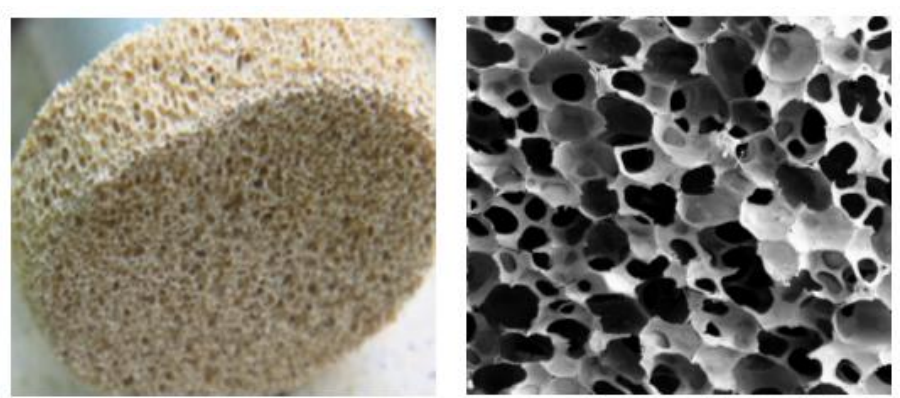

Figura 4. Derecha: Filtro cerámico con geometría circular (diámetro 25 $\mathrm{mm}$ ). Izquierda: micrografía hecha por SEM del filtro a 30 aumentos.

Otros estudios se centraron en evaluar el efecto de la concentración de sal en el proceso de adsorción, efecto de la activación de los filtros a diferentes concentraciones de ácido: $1,2,4,6$ y $8 \mathrm{~N}$ de $\mathrm{HCl}$ y $\mathrm{H}_{2} \mathrm{SO}_{4}$, y temperatura de activación, y efecto de la temperatura de calcinación en los filtros en el rango de 600 a $800^{\circ} \mathrm{C}$, de los resultados obtenidos se pudo establecer que la presencia de un electrolito $(\mathrm{NaCl})$ en la solución coloreada mejora el proceso de adsorción. El mayor valor se logró con una concentración de $30 \mathrm{~g}$ de $\mathrm{NaCl} / \mathrm{L}$ solución, reteniendo más colorante en los filtros, lo que se refleja en una menor absorbancia de la solución remanente no adsorbida.

También se pudo establecer que la activación aplicada a los filtros hace que su resistencia mecánica disminuya un poco, esto podría deberse a consecuencia de las alteraciones que la arcilla sufre en su estructura por el ataque ácido a que es sometido y que remueve parte de los aluminios que se ubican en la capa octaédrica, lo que ocasionaría un debilitamiento de la estructura.

Se comprobó que el tratamiento de activación termoácida, especialmente con soluciones $2 \mathrm{~N}$ de $\mathrm{HCl}$ y con flujo continuo, incrementan la capacidad de adsorción de los filtros a base de arcilla.

\section{Ensayos de adsorción}

Los ensayos de adsorción se llevaron a cabo con los colorantes: rojo 80 , rojo ácido $\mathrm{G}$, rhodamine $\mathrm{B}$ contenido en el efluente M4 y con el azul de metileno. Estas pruebas se realizaron colocando los filtros en una columna de vidrio por el cual se le hizo pasar la solución coloreada o el efluente M4 a un flujo constante.

Comparada con la arcilla natural, la muestra termoactivada LI-03 incrementó su poder de adsorción en 13 veces, mientras la muestra HU-01 el incremento fue de 11 veces. El proceso de adsorción se produjo a pH entre 3 y 4 y cuando fue adicionado electrolitos $(\mathrm{NaCl})$ a la solución coloreada.

Las curvas cinéticas (concentración del colorante versus tiempo), mostraron en todos los casos un comportamiento similar en el cual la concentración disminuye a medida que transcurre el tiempo en forma exponencial, se determinó que el filtro retiene la mayor cantidad del colorante en los primeros $60 \mathrm{~min}$, luego la adsorción disminuye hasta que después de aproximadamente 3 horas de contacto se llega a un punto de equilibrio donde ya no retiene más colorante.

En la figura 5 se muestra una foto de los filtros antes y después de ser probados en la adsorción del colorante 
rhodamine B contenido en el efluente M4 y en la figura 6 se observa el color de las soluciones del efluente M4 antes y después de 3 horas de contacto con los filtros de arcilla.

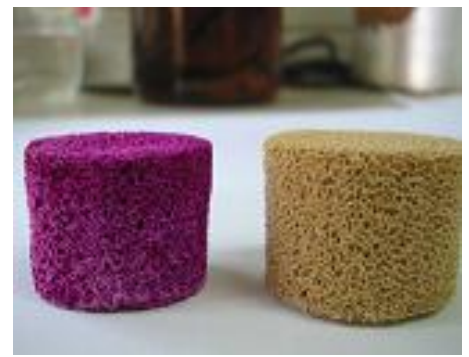

Figura 5. Fotos de los filtros. Izquierda: filtro final después de $3 \mathrm{~h}$ de contacto. Derecha: filtro inicial antes del contacto con el efluente M4.

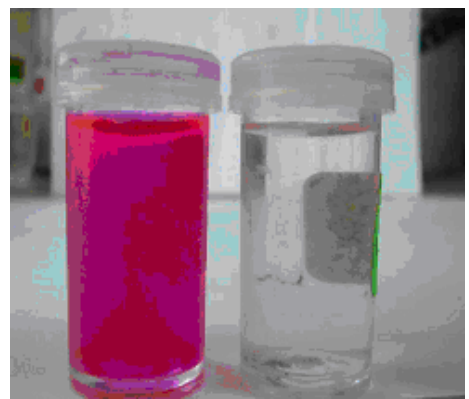

Figura 6. Fotos de las soluciones. Izquierda: Solución de efluente M4 antes del contacto con el filtro. Derecha: solución remanente de efluente M4 después de 3h de contacto con el filtro.

\section{CONCLUSIONES}

- Es posible fabricar filtros porosos a base de arcilla bentonita empleando el método de la esponja polimérica.

- Se determinó que existe un límite superior para la temperatura de sinterización de los filtros, a partir de la cual se producen cambios estructurales (formación de nuevas fases) en la arcilla, que disminuye su capacidad de adsorción.

- Con el uso de aditivos y un tratamiento térmico adecuado se pueden obtener filtros cerámicos con apariencia uniforme a menos de $600^{\circ} \mathrm{C}$. Los ensayos realizados mostraron que a pesar del calentamiento, el material no pierde su capacidad de adsorción.

- Se ha podido establecer que la presencia de sal en la solución coloreada mejora el proceso de adsorción. Se encontró que la mayor variación en la absorbancia se logra con una concentración de sal de $30 \mathrm{~g}$ de sal/L solución.

- Se comprobó que el tratamiento de activación termoácida, especialmente con soluciones $2 \mathrm{~N}$ de $\mathrm{HCl}$ y con flujo continuo, incrementan la capacidad de adsorción de los filtros a base de arcilla.

- Se encontró que la adsorción del colorante rhodamine B del efluente M4 así como del azul de metileno fue prácticamente total en los filtros (100\%). La adsorción se logró en menor grado cuando se trabajó con los colorantes rojo 80 y rojo ácido G.

- Los filtros presentaron buena resistencia mecánica cuando fueron sometidos a temperaturas mayores a $600^{\circ} \mathrm{C}$, pero esto trajo como consecuencia la disminución de su capacidad de adsorción.

\section{AGRADECIMIENTOS}

Los autores agradecen a la Dirección Académica de Investigación (Proyecto DAI-2005) de la Pontificia Universidad Católica del Perú y al CONCYTEC (Proyecto 445-2005-CONCYTEC-OAJ), por toda la ayuda y el financiamiento económico otorgados que hicieron posible la realización de este trabajo de investigación.

\section{REFERENCIAS BIBLIOGRÁFICAS}

[1] Díaz García, S. Tesis de Ingeniero Industrial. Centro de Investigación en Materiales y Metalurgia. Universidad de Ingeniería. 2002.

[2] Saggio-Woyansky, J. and Curtis E. S. Processing of porous ceramics. 1992; 71 (11): 1674-1682.

[3] Elizalde González, M.P.; Peláez Cid, A.A. Descontaminación ambiental mediante adsorbentes. Ed.Rodriguez Reynoso, F. 2005.

[4] Worrall, W. E. Clays and ceramic raw materials, $2^{\text {nd }}$ edición. 1988.

[5] Moore, D. M.; Reynolds, R. C. X-Ray diffraction, the identification and analysis of clay minerals. Ed. Oxford University Press. 1997.

[6] Bish, D.L. and Duffy, C. J. Termogravimetric Analysis of Minerals, CMS Workshop Lectures V3. Ed. The Clay Mineral Society. 2002.

[7] Schwarzwlader, K; Somers, A.V. Method of making porous ceramic articles. U.S. Patent 3.090.094, 1963.

[8] Ocelly, M.L. Catalysis Today 1988; 2: 339-345.

E-mail: msun@pucp.edu.pe 